\title{
Thioredoxin interacting protein (TXNIP) is a novel tumor suppressor in thyroid cancer
}

\author{
Jennifer A Morrison ${ }^{1 *}$, Laura A Pike', Sharon B Sams², Vibha Sharma', Qiong Zhou ${ }^{3}$ Jill J Severson', \\ Aik-Choon Tan ${ }^{4}$, William M Wood ${ }^{1}$ and Bryan R Haugen ${ }^{1}$
}

\begin{abstract}
Background: Thyroid cancer is the most common endocrine malignancy, and many patients with metastatic differentiated thyroid cancer (DTC), poorly differentiated thyroid cancer (PDTC), and anaplastic thyroid cancer (ATC) fail to respond to conventional therapies, resulting in morbidity and mortality. Additional therapeutic targets and treatment options are needed for these patients. We recently reported that peroxisome proliferator-activated receptor gamma (PPARY) is highly expressed in ATC and confers an aggressive phenotype when overexpressed in DTC cells.
\end{abstract}

Methods: Microarray analysis was used to identify downstream targets of PPARY in ATC cells. Western blot analysis and immunohistochemistry $(\mathrm{HC})$ were used to assess thioredoxin interacting protein (TXNIP) expression in thyroid cancer cell lines and primary tumor specimens. Retroviral transduction was used to generate ATC cell lines that overexpress TXNIP, and assays that assess glucose uptake, viable cell proliferation, and invasion were used to characterize the in vitro properties of these cells. An orthotopic thyroid cancer mouse model was used to assess the effect of TXNIP overexpression in ATC cell lines in vivo.

Results: Using microarray analysis, we show that TXNIP is highly upregulated when PPARY is depleted from ATC cells. Using Western blot analysis and IHC, we show that DTC and ATC cells exhibit differential TXNIP expression patterns. DTC cell lines and patient tumors have high TXNIP expression in contrast to low or absent expression in ATC cell lines and tumors. Overexpression of TXNIP decreases the growth of HTh74 cells compared to vector controls and inhibits glucose uptake in the ATC cell lines HTh74 and T238. Importantly, TXNIP overexpression in T238 cells results in attenuated tumor growth and decreased metastasis in an orthotopic thyroid cancer mouse model.

Conclusions: Our findings indicate that TXNIP functions as a tumor suppressor in thyroid cells, and its downregulation is likely important in the transition from differentiated to advanced thyroid cancer. These studies underscore the potential of TXNIP as a novel therapeutic target and prognostic indicator in advanced thyroid cancer.

Keywords: Thyroid cancer, Thioredoxin interacting protein, TXNIP, Tumor suppressor, Orthotopic model, PPARY

\section{Background}

Thyroid cancer is the most common endocrine malignancy, and it is estimated that nearly 63,000 new cases of thyroid cancer will be diagnosed in the United States in 2014 [1]. Although the majority of these patients have welldifferentiated thyroid cancer and respond favorably to conventional therapies (surgery with or without radioactive iodine $\mathrm{I}^{131}$ therapy and suppression therapy with thyroid hormone), a significant minority of patients develops

\footnotetext{
* Correspondence: Jennifer.Morrison@ucdenver.edu

'Department of Medicine, Division of Endocrinology, Diabetes, \& Metabolism, University of Colorado Anschutz Medical Campus, Aurora, Colorado, USA Full list of author information is available at the end of the article
}

advanced disease that is resistant to standard treatments. For the minority of individuals who develop anaplastic thyroid cancer (ATC), an aggressive and undifferentiated form of thyroid cancer, the prognosis is very poor and median survival is $3-5$ months [2,3]. The mechanisms underlying the development of poorly differentiated thyroid cancer (PDTC) and ATC are incompletely understood. Therapeutic options for these patients are limited, and prognosis remains dismal. This is an area for which further research and drug/therapy development is critically needed.

We recently reported that peroxisome proliferatoractivated receptor gamma (PPAR $\gamma$ ) confers an aggressive phenotype in thyroid cancer cells [4]. Nuclear PPARy 
expression was absent in differentiated thyroid cancer (DTC) cell lines and high in ATC cell lines. When PPARy was overexpressed in the DTC cell line BCPAP, in vitro proliferation and invasive capacity were increased. Furthermore, when PPAR $\gamma$ was depleted from ATC cells, in vitro proliferation and invasive capacity were inhibited and tumor growth was inhibited in two in vivo murine cancer models (an orthotopic thyroid model and a flank xenograft model). These data challenge the widelyheld assumption that PPAR $\gamma$ is a tumor suppressor and suggest that PPAR $\gamma$ may mediate the aggressive phenotype that develops as part of the transition from DTC to PDTC and ATC.

In the studies presented here, we show that TXNIP, the gene encoding thioredoxin interacting protein (TXNIP), is a negatively-regulated downstream target of PPAR $\gamma$. TXNIP is a negative regulator of cell growth and metabolism [5-11]. It modulates cellular redox status by binding to and inhibiting thioredoxin, a principal component of the cell's antioxidant system [12-14]. Furthermore, via its negative regulation of thioredoxin, TXNIP can inhibit invasion and metastasis and promote a pro-apoptotic cellular environment [15-20]. TXNIP has been shown to be a tumor suppressor in cancer [8,21-28], but its role in thyroid cells or in thyroid cancer has not been investigated. In this current study, we show that TXNIP is highly expressed in DTC and low or undetectable in ATC and appears to be a novel tumor suppressor in thyroid cancer.

\section{Results}

\section{TXNIP is upregulated in PPARy-depleted ATC cells}

We previously reported that PPAR $\gamma$ confers an aggressive phenotype in thyroid cancer cells [4]. To investigate downstream mediators of these effects, a discovery-based microarray approach was used to compare mRNA expression levels in HTh74 ATC cells expressing a PPAR $\gamma$-specific shRNA compared to a scrambled control. The most highly up- and down-regulated genes are shown in Table 1. PPARG was the most highly downregulated gene, which is consistent with the use of a PPAR $\gamma$-specific shRNA. The most highly upregulated gene in the PPAR $\gamma$ depleted HTh74 cells was thyroid peroxidase, an enzyme critical to synthesis of thyroid hormone. Interestingly, TXNIP was the second most highly upregulated gene in the PPAR $\gamma$-depleted ATC cells (>10-fold). TXNIP is a known tumor suppressor whose role in thyroid cancer has never been reported. Western blot analysis of whole cell extracts confirms that TXNIP is also upregulated at the protein level when PPAR $\gamma$ expression is knocked down in the HTh74 ATC cells (Figure 1A). These data imply that TXNIP downregulation in HTh74 cells is a downstream consequence of high nuclear PPAR $\gamma$ expression, as TXNIP expression is increased when HTh74 cells are depleted of PPAR $\gamma$. These data are consistent with a previously published report that the TXNIP promoter contains multiple PPAR $\gamma$ binding sites and that TXNIP expression is negatively regulated by binding of PPAR $\gamma$ to the TXNIP promoter and by treatment with PPAR $\gamma$ agonists [29].

\section{TXNIP is expressed at high levels in DTC cells and tissues compared with ATC, and TXNIP expression correlates inversely with glucose uptake}

We next investigated whether there is differential TXNIP expression in DTC versus ATC cells. Western blot analysis revealed high TXNIP expression in the DTC cell lines TPC-1, MDA-T41, and BCPAP in contrast to low or undetectable expression in the ATC cell lines HTh74, C643, Ocut-2, and 8505C (Figure 1B). This disparate TXNIP expression between DTC and ATC cell lines, however, was not absolute. The ATC cell lines T238 and TJH11T had levels of TXNIP expression comparable to some DTC cells, whereas the DTC cell line K1 had lower TXNIP expression in comparison to the other DTC cell lines. Similar trends were observed at the mRNA level (data not shown).

TXNIP is a known binding partner and inhibitor of the cellular antioxidant and tumor promoter thioredoxin (Trx) $[12,14]$. Thioredoxin may be localized to the cytoplasm or nucleus (Trx-1) where it can function as a cofactor, detoxify reactive oxygen species, or modulate the activity of transcription factors, or to mitochondria (Trx-2), and high Trx-1 expression is associated with more aggressive disease in other cancer types [30-36]. As TXNIP is a negative regulator of Trx and disparate TXNIP expression was observed between the panels of DTC and ATC cell lines, we sought to identify differences in Trx-1 expression patterns between the two groups. Immunoblot analysis revealed no clear differences in Trx-1 expression levels between DTC and ATC (Figure 1B).

TXNIP is also a known inhibitor of glucose uptake $[5,7,11]$. We, therefore, investigated whether differential TXNIP expression correlates with differences in glucose uptake. Analysis of glucose uptake of two ATC and two DTC cell lines was performed, and the ATC cell line HTh74, which has the lowest TXNIP levels of the examined cell lines, had the highest level of glucose uptake (Figure 1C). Interestingly, the ATC cell line T238 had glucose uptake at levels comparable to that observed with the two DTC cell lines TPC-1 and MDA-T41. This finding is consistent with a higher level of TXNIP expression in this cell line (Figure 1B).

We next investigated whether TXNIP was differentially expressed at the tumor tissue level. Immunohistochemistry to detect TXNIP protein expression was performed on paraffin-embedded tumor blocks of 13 well-differentiated primary papillary thyroid cancer (PTC) tumors and 8 ATC 
Table 1 List of highly upregulated and downregulated genes when PPARy expression is depleted from the HTh74 ATC cell line

\begin{tabular}{|c|c|c|}
\hline \multicolumn{3}{|c|}{ Gene expression changes in PPARY-depleted HTh74 cells } \\
\hline Gene & Description & Fold change \\
\hline \multicolumn{3}{|c|}{ Upregulated } \\
\hline TPO & Thyroid peroxidase & 11.7 \\
\hline TXNIP & Thioredoxin interacting protein & 10.7 \\
\hline C10rf168 & Chromosome 1 open reading frame 168 & 10.2 \\
\hline NFASC & Neurofascin homolog (chicken) & 7.6 \\
\hline SFRP4 & Secreted frizzled-related protein 4 & 7.6 \\
\hline LOC727770 & Similar to ankyrin repeat domain 20 family, member A1 & 7.2 \\
\hline ASTN1 & Astrotactin 1 & 7.1 \\
\hline \multicolumn{3}{|c|}{ Downregulated } \\
\hline PPARG & Peroxisome proliferator-activated receptor gamma & -9.2 \\
\hline$E V I 2 B$ & Ecotropic viral integration site $2 \mathrm{~B}$ & -7.5 \\
\hline LOC643201 & Hypothetical protein LOC643201 & -7.2 \\
\hline NQO1 & $\mathrm{NAD}(\mathrm{P}) \mathrm{H}$ dehydrogenase, quinone 1 & -7.1 \\
\hline ACTG2 & Actin, gamma 2, smooth muscle, enteric & -6.3 \\
\hline ARHGAP26 & Rho GTPase activating protein 26 & -6.0 \\
\hline RLN2 & Relaxin 2 & -5.7 \\
\hline
\end{tabular}

Stable cell lines were generated via transduction of the ATC cell line HTh74 with lentivirus encoding PPARY-specific shRNA or scrambled control followed by selection in puromycin $0.5 \mu \mathrm{g} / \mathrm{mL}$. Prepared RNA was then subjected to microarray analysis. Genes whose mRNA levels varied by greater than 5 -fold are shown in the table along with fold change.

patient specimens. Representative images at high and low magnification are shown for PTC (Figure 2A-B) and ATC (Figure 2C). TXNIP expression was undetectable in $63 \%$ of ATC specimens in contrast to $15 \%$ of PTC specimens, though there was one ATC specimen that exhibited high TXNIP staining. This differential TXNIP expression between PTC and ATC is similar to the pattern observed with the thyroid cancer cell lines (Figure 1B). Therefore, loss of or absence of TXNIP expression appears to correlate with more aggressive thyroid cancer in cell lines and tumor tissue.

\section{TXNIP overexpression in the ATC cells attenuates in vitro glucose uptake and growth}

Based on its differential expression in DTC and ATC, we predicted that TXNIP acts as a tumor suppressor in thyroid cells and that its downregulation plays an important role in the development of an aggressive thyroid cancer phenotype. To further investigate this hypothesis, we reexpressed TXNIP in ATC cell lines. HTh74 and T238 ATC cell lines were transduced with a retroviral vector encoding human TXNIP. Western blot analysis verified increased TXNIP expression in the transduced HTh74 (Figure 3A) and T238 (Figure 3B) cell lines compared to control cells transduced with empty vector. As a functional read out of TXNIP expression, we assessed glucose uptake in the stable cell lines. TXNIP overexpression significantly inhibited glucose uptake in both the HTh74 and
T238 cell lines (Figure 3C and 3D, respectively), consistent with its known function of glucose uptake inhibition in other tissues.

To assess the effect of TXNIP overexpression on the growth of these ATC cell lines, viable cell proliferation assays were performed under standard growth conditions. TXNIP overexpression resulted in slowed growth of HTh74 cells by 37\% (Figure 4A). Interestingly, however, TXNIP overexpression in T238 cells had no effect on the in vitro growth rate (Figure 4B). The reasons for the observed differences in TXNIP-mediated growth effects between these two cell lines are unclear, however, there are a few potential causes or factors that might contribute to this discrepancy. The baseline proliferation rate of parental T238 cells is much higher than parental HTh74 cells (data not shown), and this may obscure our ability to detect more subtle growth inhibitory effects with TXNIP overexpression in the T238 cell line. Alternatively, differential pathway activation might explain the in vitro growth differences between T238 and HTh74 cells and the ability of T238 cells to circumvent TXNIP-mediated growth deceleration. The T238 parental cell line has some basal TXNIP expression, and these cells have likely acquired the ability to resist some of the in vitro growth inhibitory effects of TXNIP via other mechanisms. In addition, the standard growth media used in propagation of these cell lines provides supplemental glutamine and glucose, and this enriched nutrient media may permit 


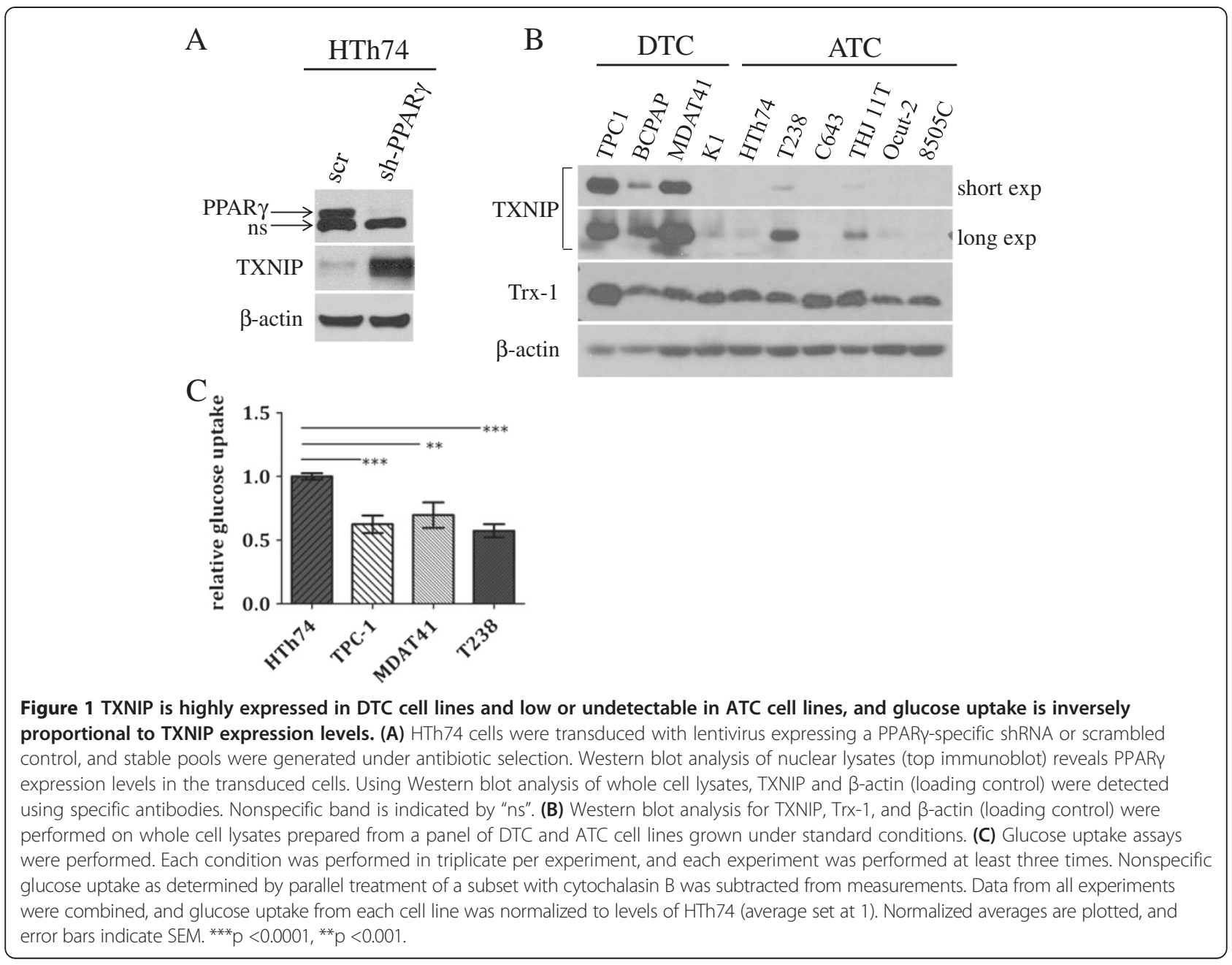

T238 cells to overcome the metabolic inhibitory effects of TXNIP overexpression in vitro. These potential limitations of in vitro assay systems, which do not adequately recapitulate in vivo tumor conditions, underscore the importance of further evaluation in an in vivo model system. In addition to proliferation assays, we performed invasion assays using an in vitro Matrigel invasion model [37], and TXNIP overexpression resulted in a trend towards decreased invasion in both cell lines but this did not reach statistical significance (Figure 4C-D).

TXNIP expression in ATC cells results in attenuated tumor growth and metastasis in an in vivo orthotopic thyroid cancer mouse model

Finally, we examined the effect of TXNIP overexpression in an in vivo orthotopic tumor model. The orthotopic thyroid cancer mouse model is a well-established model that closely mimics the features of human thyroid cancer with regard to growth and metastases than does the more commonlyused subcutaneous flank xenograft model [4,38-41]. Tumor cells expressing luciferase-IRES-GFP were injected into the right thyroid lobe and monitored weekly by IVIS imaging for tumor establishment and growth. TXNIP overexpression in the T238 cell line resulted in attenuated bioluminescence compared to vector control (Figure 5A-B), and resultant tumor volumes were significantly smaller (Figure 5C). Though there was a significant attenuation in bioluminescence as well as a trend toward smaller tumors with the TXNIP-expressing HTh74 cells compared to vector controls, the final tumor volumes were not significantly different (data not shown).

In our experience, T238 cells in this orthotopic model frequently result in lung metastases (unpublished data). To determine if TXNIP overexpression affected distant metastatic spread in this orthotopic model, lungs were collected at necropsy and snap-frozen, homogenized, and RNA was subsequently isolated and subjected to qRT-PCR to detect GFP expression, a marker of lung metastases in this model. Metastatic tumor burden was significantly reduced in the mice injected with TXNIP- 


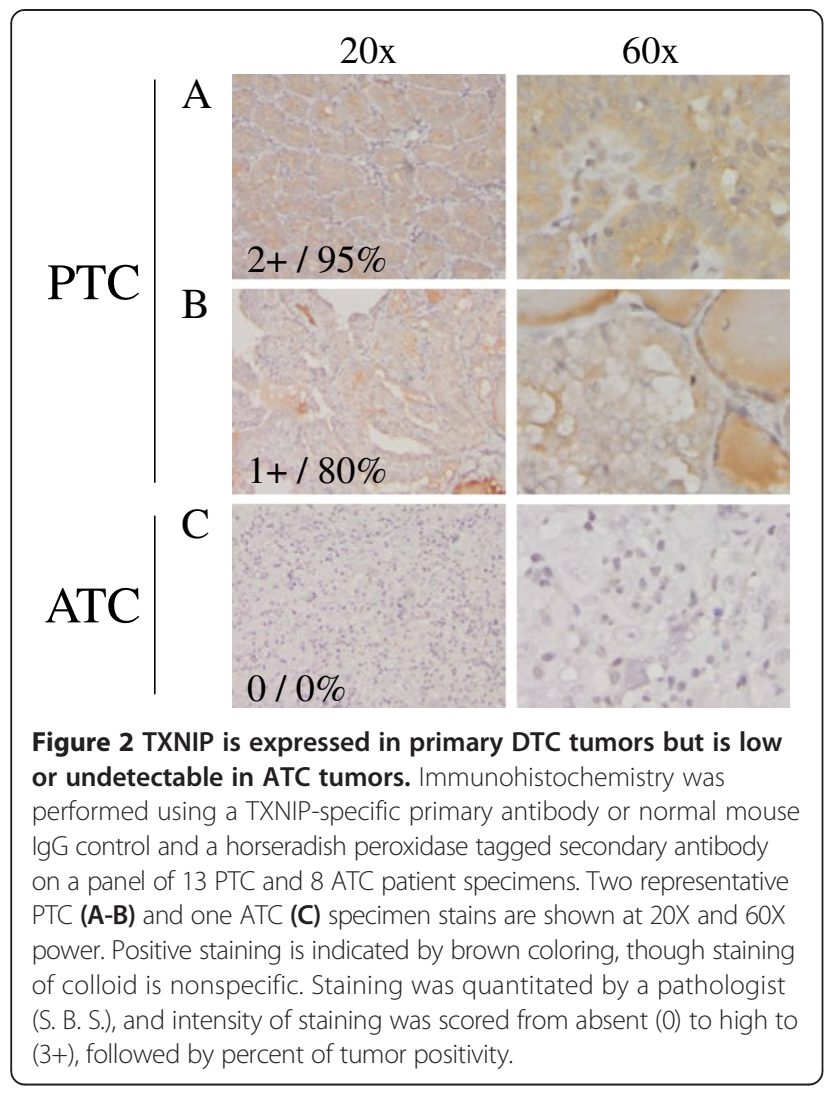

overexpressing T238 cells compared to vector controls (Figure 5D). Control mice with no tumor cells injected had no detectable GFP mRNA in their lung tissues.

\section{Discussion}

In this report, we identified TXNIP as a novel tumor suppressor in thyroid cancer. TXNIP is highly upregulated when PPAR $\gamma$, which we have previously shown to be a tumor promoter [4], is depleted from ATC cells. Furthermore, DTC cell lines and primary PTC tumors have high endogenous TXNIP levels, whereas TXNIP expression is low or absent in ATC cell lines and primary tumor specimens. This TXNIP expression pattern is opposite to what we previously reported with PPAR $\gamma$, which is highly expressed in ATC cell lines and absent in DTC cell lines and whose forced expression confers a more aggressive phenotype in thyroid cancer cells in vitro and in vivo [4]. These data are consistent with a previously published report that TXNIP is a negatively-regulated target of PPAR $\gamma$ [29]. Therefore, TXNIP expression appears to be downregulated or lost in the progression from well-differentiated thyroid tumors to more aggressive, undifferentiated tumors. TXNIP has been shown to be a tumor suppressor in other cell types, and our results here show for the first time that it serves a similar function in thyroid cells.

The novel finding that TXNIP expression is lost in the progression from well-differentiated PTC to undifferentiated, aggressive ATC is consistent with our hypothesis that TXNIP is a tumor suppressor in thyroid cancer. Patients with well-differentiated PTC respond well to conventional therapy and have an excellent overall survival rate. Poorly-differentiated PTC tumors that have lost the ability to concentrate iodine often fail to respond to conventional therapy and result in poorer outcomes, and undifferentiated ATC portends an extremely-poor prognosis and is generally fatal within $3-5$ months $[2,3]$. The apparent loss of TXNIP expression during the progression from well-differentiated to poorly-differentiated and undifferentiated thyroid cancer is consistent with its role as a tumor suppressor in thyroid cells. Loss of TXNIP expression has been reported to correlate with more aggressive disease, advanced stage, and poorer prognosis in breast, gastric, colorectal, and bladder cancers, as well as diffuse large B-cell lymphoma [23,24,42-47], and TXNIP mRNA expression has been shown to be inversely proportional to melanoma progression [27]. TXNIP expression in cancer may be downregulated through epigenetic, transcriptional, post-transcriptional, or translational mechanisms (reviewed by Zhou et al. [21]). Though the tumor promoter and cellular antioxidant Trx-1, which is inhibited by TXNIP, has been shown to confer more aggressive disease in other cancers [30-34], our data failed to show differences in Trx-1 expression levels between DTC and ATC cells.

Overexpression of TXNIP in the HTh74 ATC cell line resulted in slowed in vitro growth. This negative growth regulatory effect has been seen in other systems as well. TXNIP overexpression in the human gastric carcinoma cell lines AGS, SNU-16, and SNU-620, the promyelocytic leukemia cell line HL-60, and HTLV-I-positive T cells led to growth reduction in vitro $[8,22,48]$. Lung fibroblasts from TXNIP knockout mice proliferate at a faster rate than wild-type, implying that loss of TXNIP promotes or allows for enhanced proliferation [9]. Interestingly, we did not observe an in vitro growth inhibitory effect in the T238 cell line, which has some basal endogenous TXNIP expression. It is likely that the effects of TXNIP on cell growth and proliferation are cell-context dependent and might be circumvented through activation of alternative mitogenic pathways. Furthermore, in vitro cell culture conditions do not adequately recapitulate the tumor microenvironment and contributions of paracrinemediated signaling, underscoring the importance of in vivo studies. In accordance with this potential limitation, Goldberg and colleagues failed to see slowed in vitro growth of melanoma cells transfected with TXNIP though when injected in an orthotopic flank model in nude mice, slowed tumor growth/development was observed [27]. Although we observed a trend toward decreased invasion by TXNIP overexpression in two ATC cell lines, this effect did not reach statistical significance in our in vitro model. 

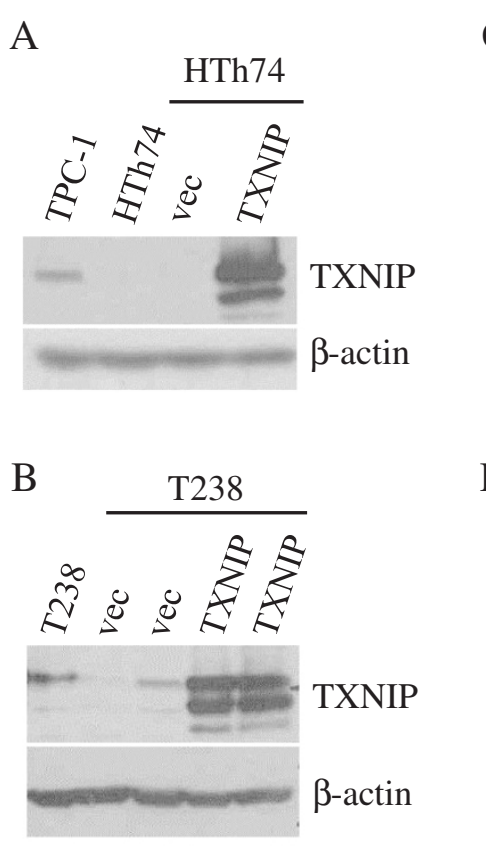

$\mathrm{D}$

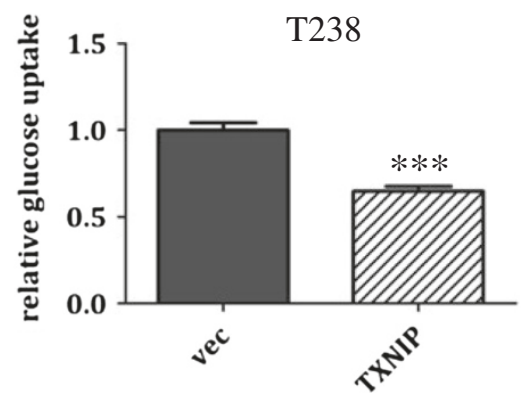

Figure 3 TXNIP overexpression in ATC HTh74 and T238 cells attenuates glucose uptake. HTh74 and T238 cells were transduced with retrovirus encoding human TXNIP or vector control as well as a selectable antibiotic resistance marker, and stable pools were generated under antibiotic selection. Western blot analysis of whole cell lysates with TXNIP- and $\beta$-actin-specific antibodies is shown for HTh74 (A) and T238 (B). Glucose uptake assays were performed as described in Figure 1 using the HTh74 stable cell lines (C) and T238 stable cell lines (D). Data from all experiments were combined, and glucose uptake from each cell line was normalized to vector control levels (average set at 1 ). ${ }^{* *} p=0.001,{ }^{* * *} p<0.0001$.

Importantly, in a well-established orthotopic murine thyroid cancer model that mimics human thyroid cancer with regard to growth and metastasis, we show that TXNIP overexpression in the ATC T238 cell line resulted in significant attenuation of both tumor growth and pulmonary metastatic burden. These data support our hypothesis that TXNIP is a tumor suppressor in thyroid cells. TXNIP has been shown to be a tumor suppressor in other animal models of cancer as well. A mouse strain with a spontaneous nonsense mutation in TXNIP has dramatically increased incidence of spontaneous hepatocellular carcinomas (HCC) [25], and TXNIP-knockout mice develop increased number and size of $\mathrm{HCC}$ in a diethylnitrosamine (DEN)-induced murine model of HCC [28]. In a murine gastric carcinoma model in which tumors are induced via infection with Helicobacter pylori and cotreatment with $N$-methyl- $N$-nitrosourea, concomitant knock out of TXNIP resulted in increased numbers of tumors, heightened preneoplastic changes, increased percentage of malignant tumors, and elevated inflammatory marker expression compared to control mice with wild-type TXNIP expression [22]. In a murine model of bladder cancer in which tumors are induced by treatment with $N$-butyl- $N$ (4-hydroxybutyl) nitrosamine (BBN), genetic deletion of TXNIP results in accelerated development of high grade and invasive tumors by $\sim 4$ weeks compared to controls with wild-type expression, however, controls eventually succumb to tumor development and TXNIP expression in these tumors has been downregulated by other mechanisms [24].

In the orthotopic ATC model, TXNIP overexpression also led to a significant reduction in pulmonary metastatic burden. Inhibition of metastasis conferred by TXNIP overexpression has been shown in other systems as well. B16F10 melanoma cells transfected with TXNIP then injected via tail vein into C57BL/6 mice resulted in decreased lung metastases [23]. TXNIP-transfected melanoma cells resulted in fewer metastases in both a nude mouse flank tumor model and IV tail injection metastasis model relative to vector controls [27]. In human breast cancer, high TXNIP levels are associated with longer metastasis-free intervals and better prognosis than those with low TXNIP expression $[43,46]$. These data implicate TXNIP as a tumor suppressor in a variety of cancers and, for the first time, is now shown to be a tumor suppressor in thyroid cells.

Curiously, TXNIP overexpression in the ATC cell line HTh74 resulted in reduced in vitro growth but no significant difference on in vivo growth in the orthotopic thyroid cancer model. Although bioluminescence signals were attenuated in the TXNIP-expressing HTh74 cells versus controls, final tumor volumes were not significantly 

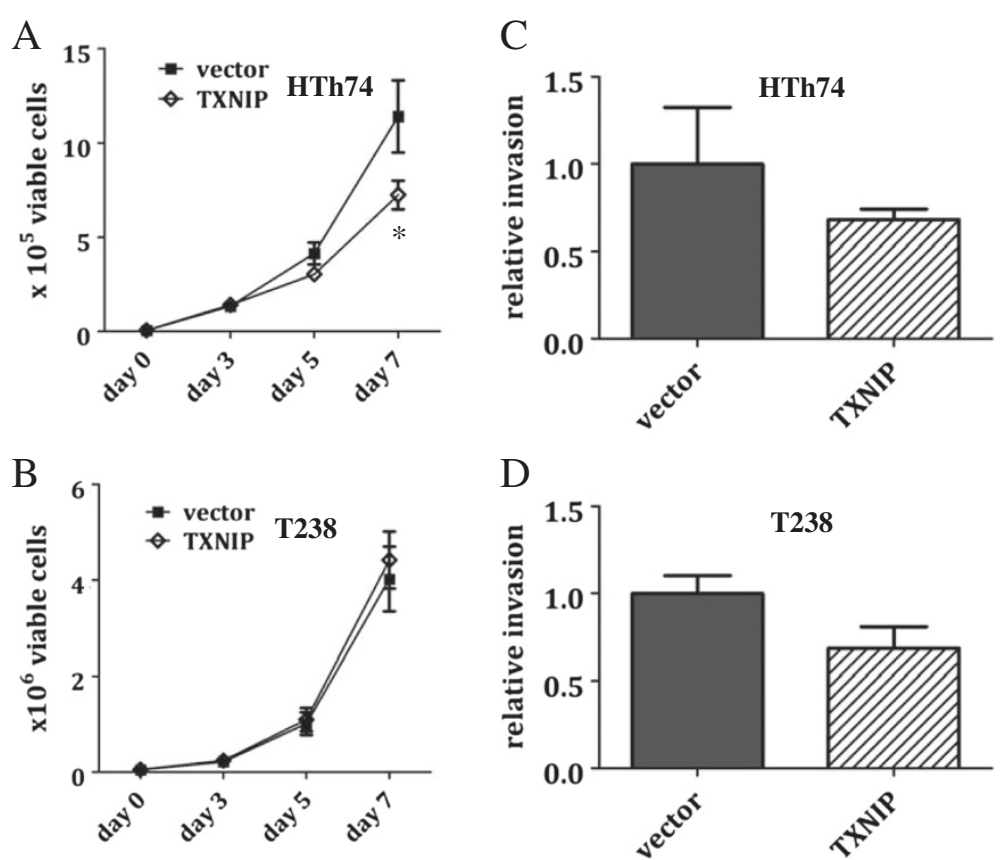

Figure 4 TXNIP overexpression inhibits in vitro growth of ATC HTh74 cells. Viable cell proliferation assays were performed for stable HTh74 (A) and T238 (B) cell lines. Briefly, 50,000 cells were plated in 6-cm plates, trypsinized and viable cell counts were determined using the ViCell automated cell counting system. Each time point was performed in duplicate, and lysates were prepared from the day 7 time point to confirm high TXNIP expression using Western blot analysis. Each experiment was performed at least three times. Mean and SEM are plotted. Closed squares ( $\mathbf{~}$ ) indicate vector control cells, and open diamonds $(\diamond)$ indicate cells with TXNIP overexpression. In vitro invasion assays were performed on the TXNIP-overexpressing stable HTh74 (C) and T238 (D) cell lines as described in the Methods section. Results from three independent experiments were combined and normalized to the vector control average and graphed with mean plus SEM. * $p<0.01$ (Figure 4A), Figure 4B $p=0.9021$, Figure $4 C p=0.3523$, Figure $4 D p=0.0754$

different, though a trend toward smaller tumors with injection of the TXNIP-expressing HTh74 cells compared to vector controls was observed. TXNIP-overexpressing and vector control tumors did not look different histologically. In our prior studies using HTh74 cells in the orthotopic murine thyroid cancer model system, we have observed that the in vivo growth rates are slower compared to other thyroid cancer cell lines (84 days to achieve $100 \mathrm{~mm}^{3}$ tumors compared with 28-35 days in other ATC cell lines). It is possible that if our study had been temporally extended, the trend in tumor volume attenuation in the TXNIP-overexpressing group might have reached statistical significance. It is also possible that TXNIP does not play a significant in vivo role on malignant behavior in the HTh74 cells, but our in vitro data would suggest otherwise.

In keeping with the known function of TXNIP as a glucose uptake inhibitor, we showed that the degree of glucose uptake was inversely correlated with TXNIP levels in the examined thyroid cancer cell lines. An interesting aspect of thyroid cancer biology relates to its properties on 2-deoxy-2-fluoro-D-glucose positron emission topography computed topography (FDG PET/CT) imaging. FDG PET imaging can be negative in many patients with
DTC and distant metastases, and this correlates with a relatively good prognosis in these patients [49,50]. Less differentiated PTC and ATC tumors are more likely to be PET positive, PET positive lesions are more likely to be resistant to conventional radioactive $\mathrm{I}^{131}$ treatment, and increased intensity of FDG uptake is associated with a poorer prognosis and increased mortality $[49,50]$. The mechanism underlying this differential glucose uptake between well-differentiated PTC and ATC is not well understood. The novel finding that TXNIP expression is low in ATC is consistent with the observed FDG uptake on PET/CT in patients with ATC, supporting a critical role for TXNIP as a metabolic regulator in thyroid cancer progression.

In addition to inducing a metabolic shift important to tumor biology, downregulation of TXNIP has other important effects in cancer cells that contribute to tumor promotion and/or progression. TXNIP can reduce tumor invasion and angiogenesis through inhibition of thioredoxin and can directly impact cell survival by promoting a pro-apoptotic environment [13,15-20]. Independent of its interaction with thioredoxin, TXNIP also has the ability to inhibit cell cycle progression by indirectly stabilizing the cell cycle inhibitor p27 ${ }^{\text {Kip1 }}$ [9]. In addition, TXNIP 

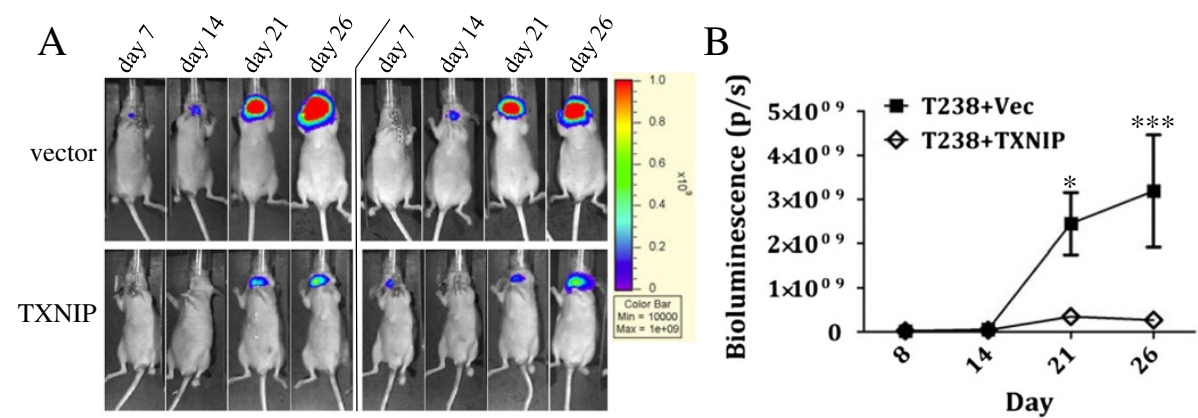

$\mathrm{C}$

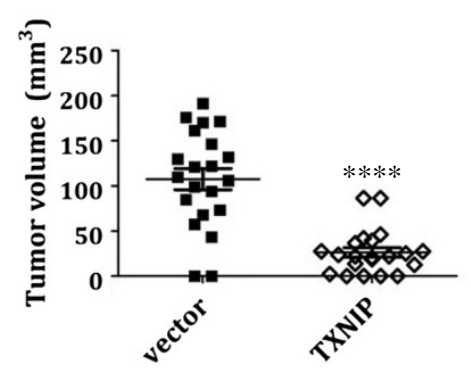

$\mathrm{D}$

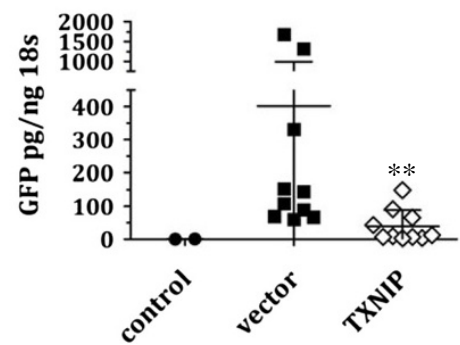

Figure 5 TXNIP overexpression in ATC T238 cells attenuates tumor growth and metastasis in an in vivo orthotopic murine thyroid cancer model. T238 cells stably expressing luciferase-IRES-GFP and either TXNIP or empty vector were injected into the right lobe of the thyroid gland of athymic nude mice with the aid of a dissecting microscope to enhance visualization. Weekly imaging with IVIS after injection of luciferin was performed to monitor tumor establishment and growth. There were 10-11 mice per group in each experiment, and the experiment was performed two times. (A) Representative images of one mouse per group imaged by IVIS over time are shown. (B) Quantitation of the bioluminescence from one experiment is shown with average and SEM. Closed squares (- indicate vector control group, and open diamonds ( () ) indicate TXNIP group. (C) Final tumor volumes, as calculated from caliper measurements, from both experiments are combined and plotted with averages and SEM. To assess for lung metastasis, RNA isolated from whole lungs was subjected to qRT-PCR analysis to assess GFP expression. Data was normalized to $18 \mathrm{~s}$ RNA levels, and averages plus SD were plotted (D). RNA isolated from lungs of mice receiving no tumor cell injections was included as an additional negative control for GFP expression and is indicated with closed circles $(\bullet) .{ }^{*} p<0.05,{ }^{* *} p=0.0021,{ }^{* * *} p<0.001,{ }^{* * *} p<0.0001$.

indirectly inhibits mTOR, a regulator of cell growth and metabolism [6]. Therefore, downregulation of TXNIP in a tumor has the potential to promote cell survival, growth, invasion, and metastasis. The exact mechanisms by which TXNIP exerts its tumor suppressive functions in thyroid cancer cells are not yet clear. Future studies of the mechanisms by which TXNIP is expressed and functions in thyroid cancer will improve our understanding of the progression to advanced thyroid cancer and help to develop more effective targeted therapies.

\section{Conclusions}

In conclusion, we report that TXNIP is a novel tumor suppressor in thyroid cancer. TXNIP is downregulated during the progression from well-differentiated thyroid cancers to poorly differentiated and anaplastic thyroid cancers. Overexpression of TXNIP in ATC cell lines resulted in slowed in vitro growth and glucose uptake inhibition. Importantly, in an in vivo orthotopic murine thyroid cancer model, TXNIP overexpression attenuated tumor growth and drastically diminished pulmonary metastatic tumor burden. These data highlight the importance of TXNIP as a potential therapeutic target and prognostic marker in advanced thyroid cancer.

\section{Methods}

\section{Cell lines and maintenance}

HTh74 and C643 cells were obtained from Dr. K. Ain (University of Kentucky, Lexington, KY) with permission from N. E. Heldin (University Hospital, Uppsala, Sweden). TPC1 cells were provided by S. Jhiang (Ohio State University, Columbus, OH). BCPAP and $8505 \mathrm{C}$ cells were provided by M. Santoro (Medical School, University of Naples Federico II, Naples, Italy). TJH11T cells were obtained from J. A. Copland (Mayo Clinic Comprehensive Cancer Center, Jacksonville, FL) and were maintained in RPMI 1640 supplemented with 10\% fetal bovine serum (FBS), non-essential amino acids, $1 \mathrm{mM}$ sodium pyruvate, $1 \mathrm{nM} \mathrm{T}, 0.5 \mu \mathrm{g} / \mathrm{mL}$ hydrocortisone, $8 \mathrm{ng} / \mathrm{mL}$ epidermal growth factor, $25 \mathrm{mM}$ HEPES, and $0.1 \mathrm{mg} / \mathrm{mL}$ Primocin. MDA-T41 cells were obtained from G. Clayman (University of Texas MD Anderson Cancer Center, Houston, TX). K1 cells were provided by D. Wynford-Thomas (Cardiff University, Cardiff, UK). T238 were obtained from L. Roque (Instituto Português de Oncologia, Lisboa, Portugal). Ocut-2 cells were obtained from N. Onoda (Osaka City University Graduate School of Medicine, Osaka, Japan). Except for TJH11T cells, all cell lines were maintained in RPMI 1640 supplemented with 5\% FBS. All cells were 
passaged at $37^{\circ} \mathrm{C}$ in $5 \% \mathrm{CO}_{2}$. Cell lines were authenticated by short tandem repeat (STR) profiling as previously described [51].

\section{PPAR $\gamma$ knockdown and microarray analysis}

PPAR $\gamma$-depleted HTh74 cells and scrambled control cells were generated as previously described using lentivirus expressing PPAR-specific shRNA or scrambled control [4]. Total RNA from PPAR $\gamma$-depleted and scrambled control cells was isolated using an RNeasy Mini Kit (Qiagen) according to the manufacturer's instructions. Integrity of the RNA preparation was verified on an Agilent Bioanalyzer 2100. Total RNA $(5 \mu \mathrm{g})$ of each cell line was used for microarray analysis using the Human Genome U133 Plus 2.0 Array (Affymetrix), performed by the Gene Expression Core of the University of Colorado Denver, Anschutz Medical Campus (Aurora, CO). Gene expression profiles were normalized by robust multichip analysis (RMA), differentially expressed genes were analyzed by fold-change, using a cut-off of 2-fold, 122 and 198 genes were found to be up and down-regulated in the knockdown line. Enrichment analysis of the gene list was performed using Database for Annotation, Visualization and Integrated Discovery (DAVID) analysis software.

\section{Western blot analysis}

Cells were trypsinized and lysed in extraction buffer (EB; 1\% Triton X-100, $10 \mathrm{mM}$ Tris pH 7.4, $5 \mathrm{mM}$ ethylenediaminetetraacetic acid (EDTA), $50 \mathrm{mM}$ sodium chloride $(\mathrm{NaCl}), 50 \mathrm{mM}$ sodium fluoride, $2 \mathrm{mM}$ sodium orthovanadate, and $1 \mathrm{X}$ cOmplete protease inhibitors [Roche Diagnostics]) and clarified by high speed centrifugation at $4^{\circ} \mathrm{C}$. For nuclear PPAR $\gamma$ and Trx-1 expression determination, cells were fractionated into nuclear and cytosolic fractions using the Active Motif Nuclear Extract system, according to the manufacturer's instructions. Whole cell and nuclear protein extracts $(25 \mu \mathrm{g})$ were diluted in Laemmli sample buffer and resolved by sodium dodecyl sulfate polyacrylamide gel electrophoresis (SDSPAGE) on $10 \%$ gels and transferred to polyvinylidene difluoride (PVDF) membranes (BioRad). Membranes were blocked for 1 hour at room temperature in 5\% nonfat dry milk in $20 \mathrm{mmol} / \mathrm{L}$ Tris $\mathrm{pH} 7.4,128 \mathrm{mmol} / \mathrm{L} \mathrm{NaCl}, 0.1 \%$ Tween 20 (TBST), then incubated overnight in primary antibody at $4^{\circ} \mathrm{C}$. Primary antibodies used for the current studies include anti-VDUP1 (TXNIP) at 1:500 (rabbit polyclonal; Invitrogen, catalog \# 403700), anti-PPAR $\gamma$ at 1:500 (rabbit polyclonal; Santa Cruz Biotechnology, catalog \# sc-7196), anti-thioredoxin-1 at 1:1,000 (C63C6 rabbit monoclonal; Cell Signaling Technology, catalog \#2429), and anti- $\beta$-actin at 1:5,000 (mouse monoclonal; Sigma-Aldrich, catalog \# A5441). After washing in TBST, membranes were incubated at room temperature for 1 hour in secondary antibodies conjugated to horseradish peroxidase (anti-rabbit at 1:5,000 for TXNIP, PPAR $\gamma$, and thioredoxin-1 blots and anti-mouse at 1:10,000 for $\beta$-actin blots; GE Healthcare). SuperSignal West Pico Chemiluminescent substrate (Thermo Scientific) was used to detect immunoreactivity. Re-Blot Plus Mild (Millipore) was used to strip blots for purposes of reprobing with an alternate primary antibody.

\section{Immunohistochemistry}

We retrospectively selected formalin-fixed, paraffinembedded blocks of primary 13 PTC and 8 ATC specimens from the University of Colorado Hospital pathology archives for analysis of TXNIP protein expression by immunohistochemistry. Institutional review board approval was obtained. Sections were deparaffinized in Histoclear, rehydrated, and antigen retrieval in $10 \mathrm{mM}$ sodium citrate buffer with $0.05 \%$ Tween 20, pH 6.0, was performed in a Biocare Medical decloaking chamber at $120^{\circ} \mathrm{C}$ for 5 minutes. Endogenous peroxidase activity was quenched by incubation in 3\% hydrogen peroxide for 30 minutes at room temperature. Tissues were blocked with $5 \%$ goat serum in phosphate-buffered saline (PBS) with $1 \%$ bovine serum albumin for 1 hour at room temperature. Slides were incubated overnight at $4^{\circ} \mathrm{C}$ in primary anti-TXNIP antibody at a concentration of 1:400 (mouse monoclonal antibody IgG1, clone JY2, MBL International, catalog \# K0205) diluted in antibody dilution buffer (0.01 M PBS, pH 7.2 with $0.05 \%$ sodium azide) or normal mouse IgG at an equivalent concentration as negative control (Santa Cruz Biotechnology, catalog \# sc-2025). Slides were incubated in secondary goat anti-mouse antibody conjugated to horseradish peroxidase diluted in PBS at a concentration of 1:400 (Dako, catalog \# P0447) for 1 hour at room temperature. For visualization, slides were incubated at room temperature for 2-4 minutes in ImmPACT 3, 3' -diaminobenzidine (DAB) peroxidase substrate (Vector Laboratories). Sections were counterstained in Mayer's hematoxylin solution, dehydrated, dried, and mounted with Cytoseal (Thermo Scientific). Stained specimens were read and score by a pathologist (S. B. Sams) based on percent of specimen that stained positively and degree of intensity ( 0 to $3+$ ).

\section{TXNIP overexpression}

A plasmid encoding human TXNIP (pcDNA3.1-hTXNIP) was a kind gift from P. Patwari (Brigham and Women's Hospital, Boston, MA). The TXNIP coding sequence was amplified via polymerase chain reaction (PCR), using primers TXNIP 1 F [5' TAG CGG CCG CAT GGT GAT GTT CAA GAA GAT CAA GT 3'] and hTXNIP EcoRI rev [5' GCG AAT TCT CAC TGC ACA TTG TTG TTG AGG A 3'], which added a NotI restriction site to the $5^{\prime}$ end and an EcoRI site on the $3^{\prime}$ end of the coding sequence, respectively. PCR product was ligated into the 
pCR2.1 shuttling vector (Invitrogen), excised via NotI and EcoRI digestion, gel purified, and directionally inserted into the retroviral vector PQCXIP (gift of S. Nordeen, University of Colorado Denver, Aurora, CO) pre-digested with NotI and EcoRI. Resultant pQCXIP-hTXNIP clones were confirmed with sequencing. Retrovirus was generated in the BOSC cell line [52] after transfection of pQCXIP or pQCXIP-hTXNIP with pCL-Ampho packaging vector (gift of H. Ford, University of Colorado Denver, Aurora, CO) using FuGENE 6 transfection reagent (Roche). Supernatant with virus was collected at 48 and 72 hours after transfection, centrifuged at low speed, filtered through $0.45 \mu \mathrm{m}$ syringe filter (Fisher Scientific), and stored at $-80^{\circ} \mathrm{C}$. Anaplastic HTh74 and T238 cells were transduced with virus-containing supernatant mixed 1:1 with growth media and supplemented with $8 \mu \mathrm{g} / \mathrm{mL}$ polybrene (Sigma-Aldrich), as previously described [4]. In the vector $\mathrm{PQCXIP}$, the coding sequence for the insert (TXNIP) is cotranscribed with a puromycin resistance gene as a bicistronic message via an internal ribosome entry site. Forty-eight hours after transduction, the cells were placed under selection in puromycin (Sigma-Aldrich) at a concentration of $0.5 \mu \mathrm{g} / \mathrm{mL}$ for HTh74 cells and $2.5 \mu \mathrm{g} / \mathrm{mL}$ for T238 cells as previously determined by kill curves.

\section{Glucose uptake assays}

Cells were grown in 12-well plates with each condition plated in triplicate. Prior to glucose uptake determination, cells were rinsed in PBS, then incubated in low glucose DMEM without serum for 4 hours at $37^{\circ} \mathrm{C}$. Cells were then incubated in Krebs buffer $(140 \mathrm{mM} \mathrm{NaCl}$, $5 \mathrm{mM}$ potassium chloride, $2.5 \mathrm{mM}$ magnesium sulfate, $1 \mathrm{mM}$ calcium chloride, $20 \mathrm{mM}$ HEPES, pH 7.4) supplemented with dimethyl sulfoxide (DMSO) or $20 \mu \mathrm{M}$ cytochalasin B (Sigma-Adrich), an actin polymerization inhibitor that blocks nonspecific glucose uptake, for 1 hour at $37^{\circ} \mathrm{C}$. Next, the cells were incubated in $0.01 \mathrm{mM}$ 2-deoxy-D-glucose [Sigma-Aldrich], $0.665 \mathrm{nCi} / \mathrm{mL}\left[1,2-{ }^{3} \mathrm{H}\right.$ ] 2-deoxy-D-glucose [PerkinElmer], and either DMSO or cytochalasin B $(20 \mu \mathrm{M})$ in Krebs buffer for an additional 20 minutes at $37^{\circ} \mathrm{C}$. After this time period, cells were immediately rinsed 3 times with ice-cold PBS, then lysed in $0.4 \mathrm{~N}$ sodium hydroxide. Base was subsequently neutralized with $0.4 \mathrm{~N}$ hydrochloric acid. Uptake of $\left[{ }^{3} \mathrm{H}\right] 2$-deoxy-D-glucose was determined by scintillation counting (Beckman Coulter). Nonspecific glucose uptake as determined by the cytochalasin B group was subtracted, and glucose uptake in pmol was normalized to protein content as determined by the BioRad DC protein assay system. Experiments were performed at least 3 times with each cell line and condition in triplicate, and data were graphed and analyzed by t-test using GraphPad Prism software.

\section{Viable cell proliferation assays}

HTh74 and T238 cells stably expressing pQCXIP vector with or without TXNIP were plated in duplicate in $6 \mathrm{~cm}$ plates at 50,000 cells/plate in RPMI 1640 supplemented with 5\% FBS, without antibiotics. At days 3, 5, and 7, cells were rinsed in PBS, incubated in $0.25 \%$ trypsin-EDTA, collected, and resuspended in RPMI with 5\% FBS. Cells were counted via the ViCell automated cell counting system. On day 7, collected cells were subsequently lysed in EB and subjected to Western blot analysis to determine TXNIP protein expression. Experiments were performed at least 3 times, and data were combined, graphed, and analyzed by 2 way ANOVA using GraphPad Prism software.

\section{Invasion assays}

Invasion assays with $2 \times 10^{5} \mathrm{HTh} 74$ and $1 \times 10^{5}$ T238 cells stably expressing QCXIP vector with or without TXNIP were performed as previously described using BD Biocoat Matrigel invasion chambers $(8 \mu \mathrm{M}$ pore size, 24 -well; BD Biosciences) [4]. Five fields per well were counted using Metamorph software (Molecular Devices), and each condition was performed in triplicate. Data from three independent experiments were combined, and data averages were normalized to the vector control mean. Statistical analysis was performed via application of the two-tailed t-test using GraphPad Prism software.

\section{Orthotopic tumor mouse model}

The right thyroid lobes of athymic nude mice were injected with 500,000 T238 QCXIP and T238 TXNIP cells stably expressing a luciferase-IRES-GFP plasmid (pEGFPLuc-N1, a kind gift from C. Li, University of Colorado Denver, Aurora, CO) in $5 \mu \mathrm{L}$ PBS as previously described [4,38-41]. Weekly bioluminescence imaging using Xenogen IVIS200 (Caliper Life Sciences) in the presence of injected luciferin substrate (Caliper Life Sciences) was performed to monitor tumor establishment and growth, and bioluminescence activity was analyzed using Living Image software (Xenogen Corporation). Bioluminescence curves were analyzed by 2-way ANOVA with Bonferroni posttests using GraphPad Prism software. There were 10-11 mice per group for each experiment, and the described experiment was performed two times. In toto, there were 21 mice in each experimental arm when data from the two independent studies were pooled. Animals were sacrificed at 26-28 days or sooner if ill or moribund, and final tumor dimensions were measured with calipers. Final tumor volumes were calculated using the formula (length $x$ width $\times$ height)/0.5236 and compared with t-test using GraphPad Prism software. All procedures were conducted in accordance with a protocol approved by the Institutional Animal Care and Use Committee of the University of Colorado Denver. 


\section{Isolation of RNA from lungs and quantitative reverse transcription polymerase chain reaction (qRT-PCR) for eGFP expression}

At time of sacrifice for the second mouse orthotopic injection experiment, lungs were collected, snap-frozen in liquid nitrogen, and stored at $-80^{\circ} \mathrm{C}$. Lungs from uninjected mice served as negative controls. To harvest RNA, lung tissues were diced in a petri dish on ice and homogenized in TRI Reagent (Sigma Aldrich) using sterile stainless steel beads and a Qiagen TissueLyser. Homogenized tissue in TRI Reagent $(1 \mathrm{~mL})$ was mixed with $200 \mu \mathrm{L}$ chloroform, centrifuged, and aqueous phase (which contains RNA) was removed. RNA purification with Qiagen RNeasy kit was then performed per the manufacturer's instructions with the exception of an added step of column incubation with RNasefree DNase I stock solution (Qiagen) in between wash steps to remove any residual DNA. RNA was ultimately eluted with RNase free water, and RNA concentration was quantitated using a Synergy H1 microplate reader (BioTek).

GFP mRNA levels were measured by real-time qRTPCR using an ABI Prism 7900 sequence detector (Applied Biosystems/Life Technologies). Primers and probe for GFP were designed with the assistance of the Prism 7900 sequence detection software (Primer Express, PE Applied Biosystems). The TaqMan probe was purchased from Life Technologies 5 ' labeled with 6-carboxyfluo rescein (FAM) and 3'-labeled with 6-caboxy-tetramethyl rhodamine (TAMRA). The forward and reverse primer sequences were GFP- F 5' - CACATGGTCCTGCTGGA GTTC- $3^{\prime}$ and GFP- R 5' - TTGTACAGCTCGTCCAT GCC- $3^{\prime}$ and the TaqMan fluorogenic probe sequence was 6FAM-CCGCCGCCGGGATCACTCT-TAMRA. Amplification reactions were performed in MicroAmp optical plates (Applied Biosystems/Life Technologies) in a $20 \mu \mathrm{l}$ mix containing 1X TaqMan Buffer A $(500 \mathrm{mM} \mathrm{KCl}$, $100 \mathrm{mM}$ Tris-HCl, $0.1 \mathrm{M}$ EDTA, $600 \mathrm{nM}$ passive reference dye ROX, $\mathrm{pH} 8.3$ at room temperature), $300 \mu \mathrm{M}$ each of dATP, dGTP, dCTP and $600 \mu \mathrm{M}$ dUTP, $5.5 \mathrm{mM}$ magnesium chloride, $900 \mathrm{nM}$ forward primer, $900 \mathrm{nM}$ reverse primer, $200 \mathrm{nM}$ probe, $1.25 \mathrm{U}$ AmpliTaq Gold DNA Polymerase and the template cDNA. Thermal cycling conditions were as follows: 2 minutes at $50^{\circ} \mathrm{C}$ followed by activation of TaqGold at $95^{\circ} \mathrm{C}$ for 10 minutes. Subsequently, 40 cycles of amplification were performed at $95^{\circ} \mathrm{C}$ for 15 seconds and $60^{\circ} \mathrm{C}$ for 1 minute. Quantities of GFP in test samples were normalized to $18 \mathrm{~s} \mathrm{r}$-RNA (PE Applied Biosystems), and the Mann Whitney test was applied to assess for statistical significance using GraphPad Prism software.

\section{Competing interests}

The authors have no competing interests to declare.

\section{Authors' contributions}

JAM, WMW, and BRH conceived of experiments outlined in this report. JAM composed the manuscript, which was approved by all authors. WMW and VS generated the HTh74 cells expressing PPARY-specific shRNA, and ACT analyzed the microarray data. JAM performed Western blot analyses, TXNIP $I H C$, subcloning of TXNIP into retroviral vector and transduction of ATC cell lines, glucose uptake assays, and viable cell proliferation assays. JAM and QZ performed the invasion assays. JAM, LAP, and QZ performed the orthotopic animal experiments. SBS provided pathology assessments of $\mathrm{HC}$ and mouse tissues. JAM and JJS isolated RNA from lungs for metastasis determination in the orthotopic mouse experiment. All authors read and approved the final manuscript.

\section{Acknowledgements}

We acknowledge the generous support of our funding sources: American Thyroid Association Research Grant Award (JAM), National Institutes of Health (NIH)/National Cancer Institute (NCI) Ruth L. Kirschstein National Research Service Award for Individual Postdoctoral Fellows F32 CA174374 (JAM), NIH/National Institute of Diabetes and Digestive and Kidney Diseases (NIDDK) Endocrinology, Diabetes, \& Metabolism Training Grant Award T32 DK007446-31 (JAM), Endocrine Fellows Foundation Endocrine Research Grant (JAM), NIH/NCI Research Grant 1R01 CA175994 (BRH, WMW), the Mary Rossick Kern and Jerome H Kern Endowment for Endocrine Neoplasms Research $(\mathrm{BRH})$, and the University of Colorado Cancer Center Gene Expression and Small Animal Imaging Cores supported by NCl Cancer Center Support Grant P30 CA46934. The content of this report is solely the responsibility of the authors and does not necessarily represent the official views of the $\mathrm{NIH}$. We thank Parth Patwari for the generous gift of the plasmid encoding human TXNIP in pcDNA3.1. We thank Isabel R. Schlaepfer and Rebecca E. Schweppe for technical advice and instruction as well as scientific discussion. We are grateful to Uma Pugazhenthi in the University of Colorado PCR core for assistance with GFP qRT-PCR and Heather Selby for assistance with the microarray analysis.

\section{Author details}

${ }^{1}$ Department of Medicine, Division of Endocrinology, Diabetes, \& Metabolism, University of Colorado Anschutz Medical Campus, Aurora, Colorado, USA. ${ }^{2}$ Department of Pathology and University of Colorado Cancer Center, University of Colorado, Aurora, Colorado, USA. ${ }^{3}$ Department of Pharmaceutical Sciences, University of Colorado Anschutz Medical Campus, Aurora, Colorado, USA. ${ }^{4}$ University of Colorado Cancer Center and Department of Medicine, Division of Medical Oncology, University of Colorado Anschutz Medical Campus, Aurora, Colorado, USA.

Received: 23 December 2013 Accepted: 13 March 2014

Published: 19 March 2014

\section{References}

1. Siegel R, Ma J, Zou Z, Jemal A: Cancer statistics, 2014. CA Cancer J Clin 2014, 64:9-29.

2. Grant CS, Thompson G: Anaplastic thyroid carcinoma: hope on the horizon? Surgery 2011, 150:1220-1221.

3. Smallridge RC, Copland JA: Anaplastic thyroid carcinoma: pathogenesis and emerging therapies. Clin Oncol (R Coll Radiol) 2010, 22:486-497.

4. Wood WM, Sharma V, Bauerle KT, Pike LA, Zhou Q, Fretwell DL, Schweppe RE, Haugen BR: PPARgamma Promotes Growth and Invasion of Thyroid Cancer Cells. PPAR Res 2011, 2011:171765.

5. Parikh H, Carlsson E, Chutkow WA, Johansson LE, Storgaard H, Poulsen $P$, Saxena R, Ladd C, Schulze PC, Mazzini MJ, Jensen CB, Krook A, Bjornholm M, Tonqvist H, Zierath JR, Ridderstrale M, Altshuler D, Lee RT, Vaag A, Groop LC, Mootha VK: TXNIP regulates peripheral glucose metabolism in humans. PLOS Med 2007, 4:e158.

6. Jin HO, Seo SK, Kim YS, Woo SH, Lee KH, Yi JY, Lee SJ, Choe TB, Lee JH, An S, Hong SI, Park IC: TXNIP potentiates Redd1-induced mTOR suppression through stabilization of Redd1. Oncogene 2011, 30:3792-3801.

7. Patwari P, Chutkow WA, Cummings K, Verstraeten VL, Lammerding J, Schreiter ER, Lee RT: Thioredoxin-independent regulation of metabolism by the alpha-arrestin proteins. J Biol Chem 2009, 284:24996-25003.

8. Han SH, Jeon JH, Ju HR, Jung U, Kim KY, Yoo HS, Lee YH, Song KS, Hwang HM, Na YS, Yang Y, Lee KN, Choi I: VDUP1 upregulated by TGF-beta1 and 1,25-dihydorxyvitamin D3 inhibits tumor cell growth by blocking cell-cycle progression. Oncogene 2003, 22:4035-4046.

9. Jeon JH, Lee KN, Hwang CY, Kwon KS, You KH, Choi I: Tumor suppressor VDUP1 increases p27(kip1) stability by inhibiting JAB1. Cancer Res 2005, 65:4485-4489. 
10. Elgort MG, O'Shea JM, Jiang Y, Ayer DE: Transcriptional and Translational Downregulation of Thioredoxin Interacting Protein Is Required for Metabolic Reprogramming during G(1). Genes Cancer 2010, 1:893-907.

11. Chutkow WA, Patwari P, Yoshioka J, Lee RT: Thioredoxin-interacting protein (Txnip) is a critical regulator of hepatic glucose production. J Biol Chem 2008, 283:2397-2406.

12. Patwari P, Higgins LJ, Chutkow WA, Yoshioka J, Lee RT: The interaction of thioredoxin with Txnip. Evidence for formation of a mixed disulfide by disulfide exchange. J Biol Chem 2006, 281:21884-21891.

13. Junn E, Han SH, Im JY, Yang Y, Cho EW, Um HD, Kim DK, Lee KW, Han PL, Rhee SG, Choi I: Vitamin D3 up-regulated protein 1 mediates oxidative stress via suppressing the thioredoxin function. J Immuno/ 2000, 164:6287-6295.

14. Nishiyama A, Matsui M, Iwata S, Hirota K, Masutani H, Nakamura H, Takagi $Y$, Sono H, Gon Y, Yodoi J: Identification of thioredoxin-binding protein-2/ vitamin $\mathrm{D}(3)$ up-regulated protein 1 as a negative regulator of thioredoxin function and expression. J Biol Chem 1999, 274:21645-21650.

15. Dunn LL, Buckle AM, Cooke JP, Ng MK: The emerging role of the thioredoxin system in angiogenesis. Arterioscler Thromb Vasc Biol 2010, 30:2089-2098

16. Liu Y, Min W: Thioredoxin promotes ASK1 ubiquitination and degradation to inhibit ASK1-mediated apoptosis in a redox activity-independent manner. Circ Res 2002, 90:1259-1266.

17. Saitoh M, Nishitoh H, Fujii M, Takeda K, Tobiume K, Sawada Y, Kawabata M, Miyazono K, Ichijo H: Mammalian thioredoxin is a direct inhibitor of apoptosis signal-regulating kinase (ASK) 1. EMBO J 1998, 17:2596-2606.

18. Welsh SJ, Bellamy WT, Briehl MM, Powis G: The redox protein thioredoxin-1 (Trx-1) increases hypoxia-inducible factor 1alpha protein expression: Trx-1 overexpression results in increased vascular endothelial growth factor production and enhanced tumor angiogenesis. Cancer Res 2002, 62:5089-5095.

19. Farina AR, Tacconelli A, Cappabianca L, Masciulli MP, Holmgren A, Beckett GJ, Gulino A, Mackay AR: Thioredoxin alters the matrix metalloproteinase/tissue inhibitors of metalloproteinase balance and stimulates human SK-N-SH neuroblastoma cell invasion. Eur J Biochem 2001, 268:405-413.

20. Oh JH, Chung AS, Steinbrenner H, Sies H, Brenneisen P: Thioredoxin secreted upon ultraviolet $A$ irradiation modulates activities of matrix metalloproteinase- 2 and tissue inhibitor of metalloproteinase- 2 in human dermal fibroblasts. Arch Biochem Biophys 2004, 423:218-226.

21. Zhou J, Yu Q, Chng WJ: TXNIP (VDUP-1, TBP-2): a major redox regulator commonly suppressed in cancer by epigenetic mechanisms. Int J Biochem Cell Biol 2011, 43:1668-1673.

22. Kwon HJ, Won YS, Nam KT, Yoon YD, Jee H, Yoon WK, Nam KH, Kang JS, Han SU, Choi IP, Kim DY, Kim HC: Vitamin D(3) upregulated protein 1 deficiency promotes N-methyl-N-nitrosourea and Helicobacter pylori-induced gastric carcinogenesis in mice. Gut 2012, 61:53-63.

23. Shin D, Jeon JH, Jeong M, Suh HW, Kim S, Kim HC, Moon OS, Kim YS, Chung JW, Yoon SR, Kim WH, Choi I: VDUP1 mediates nuclear export of HIF1alpha via CRM1-dependent pathway. Biochim Biophys Acta 2008, 1783:838-848.

24. Nishizawa K, Nishiyama H, Matsui Y, Kobayashi T, Saito R, Kotani H, Masutani H, Oishi S, Toda Y, Fujii N, Yodoi J, Ogawa O: Thioredoxin-interacting protein suppresses bladder carcinogenesis. Carcinogenesis 2011, 32:1459-1466

25. Sheth SS, Bodnar JS, Ghazalpour A, Thipphavong CK, Tsutsumi S, Tward AD Demant $\mathrm{P}$, Kodama T, Aburatani H, Lusis AJ: Hepatocellular carcinoma in Txnip-deficient mice. Oncogene 2006, 25:3528-3536.

26. Shin $\mathrm{KH}, \mathrm{Kim} \mathrm{RH}$, Kang MK, Park NH: hnRNP G elicits tumor-suppressive activity in part by upregulating the expression of Txnip. Biochem Biophys Res Commun 2008, 372:880-885.

27. Goldberg SF, Miele ME, Hatta N, Takata M, Paquette-Straub C, Freedman LP, Welch DR: Melanoma metastasis suppression by chromosome 6: evidence for a pathway regulated by CRSP3 and TXNIP. Cancer Res 2003, 63:432-440

28. Kwon HJ, Won YS, Suh HW, Jeon JH, Shao Y, Yoon SR, Chung JW, Kim TD, Kim HM, Nam KH, Yoon WK, Kim DG, Kim JH, Kim YS, Kim DY, Kim HC, Choi I: Vitamin D3 upregulated protein 1 suppresses TNF-alpha-induced NF-kappaB activation in hepatocarcinogenesis. J Immuno/ 2010, 185:3980-3989.
29. Qi W, Chen X, Holian J, Tan CY, Kelly DJ, Pollock CA: Transcription factors Kruppel-like factor 6 and peroxisome proliferator-activated receptor\{gamma\} mediate high glucose-induced thioredoxin-interacting protein. Am J Pathol 2009, 175:1858-1867.

30. Ogata FT, Batista WL, Sartori A, Gesteira TF, Masutani H, Arai RJ, Yodoi J, Stern A, Monteiro HP: Nitrosative/Oxidative Stress Conditions Regulate Thioredoxin-Interacting Protein (TXNIP) Expression and Thioredoxin-1 (TRX-1) Nuclear Localization. PLoS One 2013, 8:e84588.

31. Nagano M, Hatakeyama K, Kai M, Nakamura H, Yodoi J, Asada Y, Chijiiwa K: Nuclear expression of thioredoxin-1 in the invasion front is associated with outcome in patients with gallbladder carcinoma. HPB (Oxford) 2012, 14:573-582.

32. Shan W, Zhong W, Zhao R, Oberley TD: Thioredoxin 1 as a subcellular biomarker of redox imbalance in human prostate cancer progression. Free Radic Biol Med 2010, 49:2078-2087.

33. Lincoln DT, Ali Emadi EM, Tonissen KF, Clarke FM: The thioredoxinthioredoxin reductase system: over-expression in human cancer. Anticancer Res 2003, 23:2425-2433.

34. Cha MK, Suh KH, Kim IH: Overexpression of peroxiredoxin I and thioredoxin1 in human breast carcinoma. J Exp Clin Cancer Res 2009, 28:93.

35. Arner ES, Holmgren A: Physiological functions of thioredoxin and thioredoxin reductase. Eur J Biochem 2000, 267:6102-6109.

36. Yoshihara E, Masaki S, Matsuo Y, Chen Z, Tian H, Yodoi J: Thioredoxin/ Txnip: Redoxisome, as a Redox Switch for the Pathogenesis of Diseases. Front Immunol 2014, 4:514.

37. Hendrix MJ, Seftor EA, Seftor RE, Fidler IJ: A simple quantitative assay for studying the invasive potential of high and low human metastatic variants. Cancer Lett 1987, 38:137-147.

38. Chan CM, Jing X, Pike LA, Zhou Q, Lim DJ, Sams SB, Lund GS, Sharma V, Haugen BR, Schweppe RE: Targeted inhibition of Src kinase with dasatinib blocks thyroid cancer growth and metastasis. Clin Cancer Res 2012, 18:3580-3591

39. Kim S, Park YW, Schiff BA, Doan DD, Yazici Y, Jasser SA, Younes M, Mandal M, Bekele BN, Myers JN: An orthotopic model of anaplastic thyroid carcinoma in athymic nude mice. Clin Cancer Res 2005, 11:1713-1721.

40. Ahn SH, Henderson Y, Kang Y, Chattopadhyay C, Holton P, Wang M, Briggs K, Clayman GL: An orthotopic model of papillary thyroid carcinoma in athymic nude mice. Arch Otolaryngol Head Neck Surg 2008, 134:190-197.

41. Nucera C, Nehs MA, Mekel M, Zhang X, Hodin R, Lawler J, Nose V, Parangi S: A novel orthotopic mouse model of human anaplastic thyroid carcinoma. Thyroid 2009, 19:1077-1084.

42. Woolston CM, Madhusudan S, Soomro IN, Lobo DN, Reece-Smith AM, Parsons SL, Martin SG: Thioredoxin interacting protein and its association with clinical outcome in gastro-oesophageal adenocarcinoma. Redox Bio/ 2013, 1:285-291.

43. Woolston CM, Zhang L, Storr SJ, Al-Attar A, Shehata M, Ellis IO, Chan SY, Martin SG: The prognostic and predictive power of redox protein expression for anthracycline-based chemotherapy response in locally advanced breast cancer. Mod Pathol 2012, 25:1106-1116.

44. Lim JY, Yoon SO, Hong SW, Kim JW, Choi SH, Cho JY: Thioredoxin and thioredoxin-interacting protein as prognostic markers for gastric cancer recurrence. World J Gastroenterol 2012, 18:5581-5588.

45. Ikarashi M, Takahashi Y, Ishii Y, Nagata T, Asai S, Ishikawa K: Vitamin D3 up-regulated protein 1 (VDUP1) expression in gastrointestinal cancer and its relation to stage of disease. Anticancer Res 2002, 22:4045-4048.

46. Cadenas C, Franckenstein D, Schmidt M, Gehrmann M, Hermes M, Geppert B, Schormann W, Maccoux LJ, Schug M, Schumann A, Wilhelm C, Freis E, Ickstadt K, Rahnenfuhrer J, Baumbach Jl, Sickmann A, Hengstler JG: Role of thioredoxin reductase 1 and thioredoxin interacting protein in prognosis of breast cancer. Breast Cancer Res 2010, 12:R44.

47. Tome ME, Johnson DB, Rimsza LM, Roberts RA, Grogan TM, Miller TP, Oberley LW, Briehl MM: A redox signature score identifies diffuse large B-cell lymphoma patients with a poor prognosis. Blood 2005, 106:3594-3601.

48. Nishinaka Y, Nishiyama A, Masutani H, Oka S, Ahsan KM, Nakayama Y, Ishii Y, Nakamura H, Maeda M, Yodoi J: Loss of thioredoxin-binding protein-2/ vitamin D3 up-regulated protein 1 in human T-cell leukemia virus type 
I-dependent T-cell transformation: implications for adult T-cell leukemia leukemogenesis. Cancer Res 2004, 64:1287-1292.

49. Wang W, Larson SM, Fazzari M, Tickoo SK, Kolbert K, Sgouros G, Yeung H, Macapinlac H, Rosai J, Robbins RJ: Prognostic value of [18F]

fluorodeoxyglucose positron emission tomographic scanning in patients with thyroid cancer. J Clin Endocrinol Metab 2000, 85:1107-1113.

50. Deandreis D, Al Ghuzlan A, Leboulleux S, Lacroix L, Garsi JP, Talbot M, Lumbroso J, Baudin E, Caillou B, Bidart JM, Schlumberger M: Do histological, immunohistochemical, and metabolic (radioiodine and fluorodeoxyglucose uptakes) patterns of metastatic thyroid cancer correlate with patient outcome? Endocr Relat Cancer 2011, 18:159-169.

51. Schweppe RE, Klopper JP, Korch C, Pugazhenthi U, Benezra M, Knauf JA, Fagin JA, Marlow LA, Copland JA, Smallridge RC, Haugen BR:

Deoxyribonucleic acid profiling analysis of 40 human thyroid cancer cell lines reveals cross-contamination resulting in cell line redundancy and misidentification. J Clin Endocrinol Metab 2008, 93:4331-4341.

52. Pear WS, Nolan GP, Scott ML, Baltimore D: Production of high-titer helper-free retroviruses by transient transfection. Proc Natl Acad Sci U S A 1993, 90:8392-8396.

doi:10.1186/1476-4598-13-62

Cite this article as: Morrison et al:: Thioredoxin interacting protein (TXNIP) is a novel tumor suppressor in thyroid cancer. Molecular Cancer 2014 13:62.

\section{Submit your next manuscript to BioMed Central and take full advantage of:}

- Convenient online submission

- Thorough peer review

- No space constraints or color figure charges

- Immediate publication on acceptance

- Inclusion in PubMed, CAS, Scopus and Google Scholar

- Research which is freely available for redistribution 